\title{
Fault Feature Extraction of Bearing Fault in Wind Turbine Generator Based on the Variational Modal Decomposition and Spectral Kurtosis
}

\author{
ShuangWei Guo ${ }^{1, a}$, Wenmin Zhang ${ }^{2}$, Hongshan Zhao ${ }^{3}$ \\ ${ }^{1}$ Electrical and Electronic Engineering Institute, North China Electric Power University, Baoding \\ 071003, Hebei Province, China. \\ ${ }^{2}$ Electric Power Maintenance Company of GanSu Province, Wenmin Zhang \\ ${ }^{3}$ Electrical and Electronic Engineering Institute, North China Electric Power University, Baoding \\ 071003, HebeiProvince, China. \\ ${ }^{a} E m a i l: 1262589712 @ q q . c o m$
}

Keywords: Variational mode decomposition; Spectral Kurtosis; Wind turbine generator; Fault feature extraction.

Abstract: In order to extract fault features of rolling bearing effectively, a method which is based on variational mode decomposition and spectral kurtosis was proposed. The Intrinsic Mode Function (IMF) is obtained by variational mode decomposition.The intrinsic mode function (IMF) is obtained by variational mode decomposition. The IMF containing fault information was selected to reconstructed based on the spectral kurtosis and then extracting the fault feature frequency by envelope spectrum analysis and good results are obtained.

\section{Introduction}

The bearing is the key part of the mechanical transmission system and the generator system, which plays a vital role in the process of power transmission ${ }^{[1]}$.If the bearing fails, the normal operation of the wind turbine transmission system may be affected, even resulting in the stop of the machine. In this paper, a novel method for bearing fault feature extraction is proposed based on variational mode decomposition(VMD) and kurtosis index. By VMD, the fault signal is decomposed into a number of IMFs and the signal is reconstructed based on the spectral kurtosis. The signal is then are demodulated by the envelope analysis and the fault characteristics of the bearing are extracted.

\section{Theoretical background}

Variational mode decomposition (VMD). Variational mode decomposition (VMD) ${ }^{[3]}$ is a new method for signal decomposition estimation. In the VMD algorithm, each intrinsic mode function (IMF) can be regarded as an amplitude modulated signal:

$$
u_{k}(t)=A_{k}(t) \cos \left(\phi_{k}(t)\right)
$$

where $\omega_{k}(t)=\phi_{k}^{\prime}(t)=d \phi_{k}(t) / d t, A_{k}(t)$ is the instantaneous amplitude of the signal, $\omega_{k}(t)$ is the instantaneous frequency. Compared with $\phi_{k}(t), A_{k}(t)$ and $\omega_{k}(t)$ change slowly. That is to say, in the range of $[t-\delta, t+\delta]\left(\delta=2 \pi / \phi_{k}^{\prime}(t)\right), u_{k}(t)$ can be regarded as a harmonic signal, whose amplitude is $A_{k}(t)$, frequency is $\omega_{k}(t)$.

The decomposition process of VMD is actually a variational problem solving process. Assuming that each IMF has a limited bandwidth, the variational problem can be expressed as to find $k$ different intrinsic mode function(IMF) to minimize the sum of the estimated bandwidth and the sum of IMF is the original input signal $f$. Specific steps are as follows:

(1) For each IMF, the single side spectrum is obtained by calculating the corresponding analytical signal with the Hilbert transform: $(\delta(t)+j / \pi t) * u_{k}(t)$.

(2) By adding an exponential term $e^{-j \omega_{k} t}$ to adjust the estimated center frequency of each analytical signals corresponding to the independent feature components, the frequency spectrum of each mode is shifted to the base band: $\left[(\delta(t)+j / \pi t) * u_{k}(t)\right] e^{-j \omega_{k} t}$. 
(3) Analysis the bandwidth of the demodulated signal with the H1 Gaussian smoothness i.e.the squared L2-norm of the gradient, The resulting constrained variational problem is the following:

$$
\begin{gathered}
\min \left\{\sum_{k} \square \partial t\left[(\delta(t)+j / \pi t) * u_{k}(t)\right] e^{-j \omega_{k} t} \square_{2}^{2}\right\} \\
\text { s.t } \sum u_{k}=f
\end{gathered}
$$

where $\left\{\omega_{k}\right\}:=\left\{\omega_{1}, \omega_{2}, \cdots \omega_{k}\right\},\left\{u_{k}\right\}:\left\{u_{1}, u_{2}, \cdots u_{k}\right\}$.

(4) Two penalty factor $\alpha$ and Lagrange multiplier operator $\lambda(t)$ are introduced to construct the extended Lagrange expression $\Gamma\left(u_{k}, \omega_{k}, \lambda\right)$

$$
\begin{gathered}
\Gamma\left(u_{k}, \omega_{k}, \lambda\right)=\alpha \sum_{k} \square \partial t\left[(\delta(t)+j / \pi t) * u_{k}(t)\right] e^{-j \omega_{k} t} \square_{2}^{2} \\
+\square f-\sum u_{k} \square_{2}^{2}+\left\langle\lambda, f-\sum u_{k}\right\rangle
\end{gathered}
$$

Spectral Kurtosis. General vibration signal model of rolling bearings can be expressed by the following formula:

$$
Z(t)=X(t)+N(t)
$$

where $Z(t)$ is the actual measurement of the vibration signal; $X(t)$ is the detected fault signal; $N(t)$ is strong additive noise.

Spectral kurtosis ${ }^{[4]}$ is defined as the 4 order cumulant spectrum energy normalization, the formula is as follows:

$$
K_{Z}(f)=K_{X}(f) /[1+\rho(f)]^{2}
$$

Where $K_{Z}(f)$ is the spectral kurtosis of the $X(t) . \rho(f)$ is the signal to noise ratio. $\rho(f)=S_{N}(f) / S_{x}(f), S_{N}(f)$ and $S_{x}(f)$ are respectively power spectral density of noise and signal.

$K_{Z}(f)$ is approximately equal to the $K_{Z}(f)$ in the frequency where the signal to noise ratio is very high, however, $K_{Z}(f)$ tends to be zero where the noise is very strong. So the frequency bands containing fault signals could be detected with the spectral kurtosis.

\section{Steps of the algorithm}

The steps of the algorithm are displayed as follows:

(1) The original vibration signal is decomposed to IMF by VMD.

(2) Computing the spectral kurtosis of the different IMFs, choose the suitable IMFs to reconstruct the signal, which contain fault information.

(3) Making an envelope analysis of the signal obtained from step 2 to determine whether there has a failure and to find out the location of the fault.

\section{The result of the analysis}

In this paper, the fault data comes from the bearing data center of Case Western Reserve University

The time domain and frequency domain of the vibration signal of the bearing outer ring fault is shown in Figure 1 (a) and 1 (b). The signal sampling frequency is $12000 \mathrm{~Hz}$ and the number of sampling points is 8000 , the outer ring fault characteristic frequency is $106.5 \mathrm{~Hz}$.
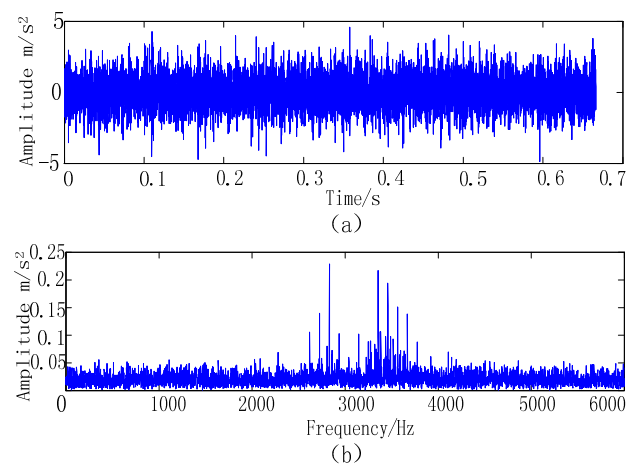

Fig.1 Time domain and frequency domain diagram of fault signal of bearing outer ring 
The original non-stationary vibration signal is decomposed by VMD and five IMF components are obtained. Different IMF contains different frequency components, and each IMF is closely around a central frequency. After calculation, the spectral kurtosis value of the IMF component is shown in Table 1.

Tab.1 The spectral kurtosis of the IMF after the VMD decomposition

\begin{tabular}{lccccc} 
& IMF1 & IMF2 & IMF3 & IMF4 & IMF5 \\
\hline $\begin{array}{l}\text { Spectral } \\
\text { Kurtosis }\end{array}$ & 0.913 & 2.553 & 2.821 & 0.871 & 0.962
\end{tabular}

From table 1, it can be seen that the spectral kurtosis value of the IMF2 and IMF3 larger, therefore, signal is reconstructed with the IMF2 and IMF3, the time domain and frequency domain of the reconstructed signal is shown in Figure 2.
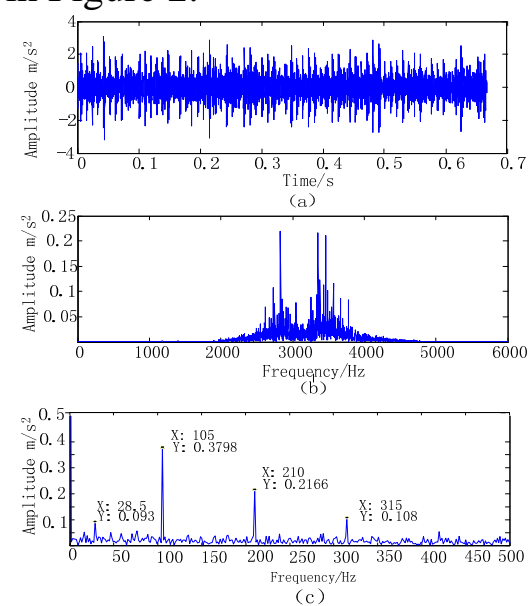

Fig.2 Time domain and frequency domain and envelope spectrum of signal of

the reconstructed signal of the outer ring fault

The rotating speed of the rotating shaft is $1796 \mathrm{r} / \mathrm{min}$, and the frequency is $29.63 \mathrm{~Hz}$. In Figure 2 (c), a peak can be seen at the frequency of $28.5 \mathrm{~Hz}$, which corresponds to the theoretical transfer frequency. At the same time, a distinct peak can be seen in the $105 \mathrm{~Hz}$,corresponding to the fault characteristic frequency of the bearing outer ring. What's more, the frequency components of the $210 \mathrm{~Hz}$ and $315 \mathrm{~Hz}$ have obvious peak value, which correspond to the fault characteristic frequency of two and three times frequency, and meets the fault characteristic of the bearing outer ring.

Using the same method to deal with the inner ring fault signal, the time domain and frequency domain are shown in Figure 3 (a) and 3 (b).At this point, the drive end of the transfer frequency is $1794 \mathrm{r} / \mathrm{min}$, the theoretical frequency of $29.9 \mathrm{~Hz}$, after the calculation of the fault characteristic frequency of $160 \mathrm{~Hz}$.Using the above method, the signal envelope spectrum of signal processing is shown in Figure 3 (c).
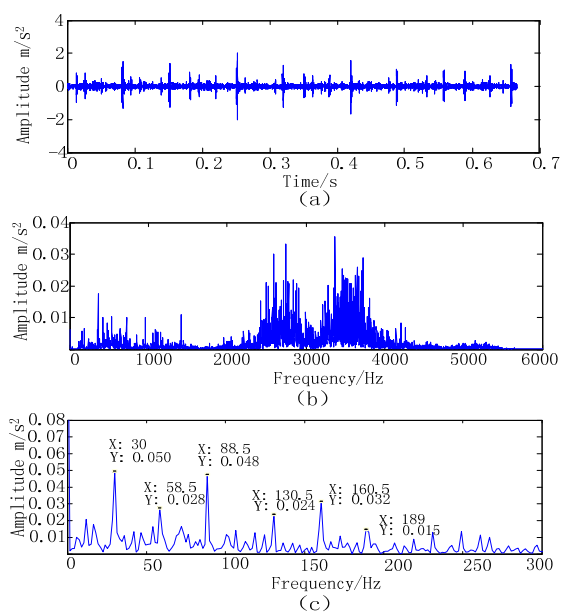

Fig.3 Time domain and frequency domain Signal time domain frequency domain and envelope spectrum of signal of the reconstructed signal of the inner ring fault 
In Figure 3 (c), it is seen that the peak value of $30 \mathrm{~Hz}, 58.5 \mathrm{~Hz}$, and $88.5 \mathrm{~Hz}$ are obvious, which is corresponding to the frequency of two and three times. In the $160.5 \mathrm{~Hz}$,the obvious peaks can also be seen, corresponding to the fault characteristic frequency. Moreover, the $189 \mathrm{~Hz}$ and $130.5 \mathrm{~Hz}$ in the vicinity of the $160.5 \mathrm{H}$ have a more obvious projection, which corresponds to 1 times of the frequency band, and meet the fault characteristics of the bearing inner ring.

\section{Conclusion}

In this paper, the independent feature component is obtained and the independent feature component are selected by the spectral kurtosis, and the problem of the optimal bands selection is solved effectively .The fault characteristics of the signal are extracted from the noise reduction, and the good results are obtained.

\section{Acknowledgements}

This work was financially supported by the National Natural Science Foundation of China (51277074)

\section{Reference}

[1] Chen Xuefen, Li Jimeng, Cheng Hang, Li Bing, et al. Research and application of condition monitoring and fault diagnosis technology in wind turbines [J].Power System Technology ,2011,47(9):8-22.

[2] Zhou Zhi, Zhu Yongsheng, Zhang Youyun, Zhu Chuanfeng, Wang Peng , et al. Adaptive fault diagnosis of rolling bearings based on EEMD and demodulated resonance [J]. Journal of Vibration and Shock, 2013, 32(2):6-88.

[3] DragomiretskiyK,Zosso D. Variational mode decomposition[J]. IEEE Tran on Signal Processing, 2014, 62(3):31-544.

[4] Antoni J. The spectral kurtosis: A useful tool for characterising non-stationary signals[ J]. MechanicalSystems and SignalProcessing,2006, 20: 282- 307. 